Research Paper

\title{
Linc-KILH potentiates Notch1 signaling through inhibiting KRT19 phosphorylation and promotes the malignancy of hepatocellular carcinoma
}

\author{
Xudong Zhang1\#, Xiaoliang Xu'2,3\#, Zechuan Zhang3\#, Cailin Xue1, Zhijun Kong1, Siyuan Wu1, Xiao Yun1, \\ Yue $\mathrm{Fu}^{1}$, Chunfu Zhu ${ }^{1 凶}$ and Xihu Qin ${ }^{1 凶}$ \\ 1. The Affiliated Changzhou NO.2 People's Hospital of Nanjing Medical University, 29 XingLongXiang Road, Changzhou, Jiangsu 213000, P.R. China. \\ 2. School of medicine, Southeast University, Nanjing, China. \\ 3. Department of Hepatobiliary Surgery of Nanjing Drum Tower Hospital, Nanjing Medical University, Nanjing, China. \\ \#These authors contributed equally to this work. \\ $\triangle$ Corresponding authors: Xihu Qin and/or Chunfu Zhu, The Affiliated Changzhou NO.2 People's Hospital of Nanjing Medical University, 29 XingLongXiang \\ Road, Changzhou, Jiangsu 213000, P.R. China.
}

() The author(s). This is an open access article distributed under the terms of the Creative Commons Attribution License (https://creativecommons.org/licenses/by/4.0/). See http://ivyspring.com/terms for full terms and conditions.

Received: 2020.08.22; Accepted: 2021.01.16; Published: 2021.02.08

\begin{abstract}
Long noncoding RNAs (LncRNAs) are emerging as crucial regulators in the pathophysiological process of various tumors, including HCC. Here, we identify a novel IncRNA Linc-KILH (KRT19 interacting long noncoding RNA in hepatocellular carcinoma), which is significantly up-regulated in HCC tissues and positively correlated with larger tumor size, severer microvascular invasion, more intrahepatic metastasis and decreased survival of HCC patients. Silence of Linc-KILH remarkably inhibited the proliferation and metastasis abilities of KRT19-positive HCC cells in vitro and in vivo. Mechanistically, Linc-KILH interacts with KRT19 and then inhibits the phosphorylation of KRT19 on Ser35, thereby, enhancing the translocation of KRT19 from cytoplasm to membrane in KRT19 positive HCC cells. Additionally, we validated that KRT19 interacts with $\beta$-catenin but not RACl in HCC cells. Linc-KILH enhanced the interaction between $\beta$-catenin and KRT19 in cytoplasm and promoted the nuclear translocation of $\beta$-catenin in HCC cells. Furthermore, Linc-KILH could enhance the promoting function of KRT19 on Notchl signaling with the existence of KRT19 in HCC cells. Collectively, we revealed that Linc-KILH exerts a vital function in KRT19 positive HCC progression and may likely be developed into an effective therapeutic target for HCC.
\end{abstract}

Key words: hepatocellular carcinoma, microvascular invasion, long noncoding RNAs

\section{Introduction}

As a highly lethal cancer, it is estimated that hepatocellular carcinoma (HCC) causes almost half a million deaths annually in East Asia and South Africa [1]. Although continuous advances in HCC treatment (for example, surgical resection, liver transplantation, radiation therapy, targeted chemotherapy and molecular-targeted therapy) have been developed, the long-term survival rate of HCC patients still remains low $[2,3]$. Therefore, there is an urgent need to further comprehensively explore the underlying molecular mechanisms of HCC and identify novel prognostic and therapeutic targets.
KRT19 (Cytokeratin 19), one of the smallest members of the acidic type I cytokeratin family proteins (KRTs), has been reported as widely detectable tumor maker in various cancers, including HCC [4-7]. Several studies have revealed that KRT19 can function as a marker of poor prognosis in HCC [8-10]. For instance, knockdown of KRT19 suppress the progression of HCC and its stem cell properties through regulating TGF $\beta /$ Smad and Notch1 signaling pathways [5]. Additionally, a recent study revealed that activation of PDGFR alpha/laminin B1/KRT19 signaling pathway promotes the 
progression of HCC through developing microvascular invasion, metastasis and early recurrence in HCC [11]. As a cytoplasmic intermediate filament protein, KRT19 can regulate diverse properties in various cancers through interacting with a variety of signal transduction molecules. Recent studies shown that KRT19 could function as a differential modulator of Wnt/ $\beta$ Catenin/Notch signaling and resulted in opposing regulation of cancer properties in breast and colon cancers [6, 7]. Thus, extensive molecular researches on the specific functional regulation of KRT19 in HCC need to be further elucidated.

Long noncoding RNAs (LncRNAs) are traditionally identified as transcripts longer than 200nt and with no or limited protein coding ability [12]. Accumulating researches have revealed that LncRNAs are regularly dysregulated and closely correlated with the development and outcome of various cancers, including HCC $[13,14]$. Up to now, the researches of liver cancer related LncRNAs is still in the development stage, more efforts should be put into the exploration of functional LncRNAs in HCC. Among the various mechanisms of LncRNAs, one of the most interesting way is to participate in the regulation of downstream signaling pathways through the interaction with binding proteins [15-18]. For instance, a recent study revealed a lncRNA interacting with STAT3 and then regulating its phosphorylation, thus modulated STAT3 signal transduction in dendritic cells [19]. Here, we proposed that further identification and functional elucidation of KRT19 associated LncRNAs could provide new insights into KRT19-mediated tumor promotion in HCC. However, whether or not LncRNAs could participate in KRT19 associated signaling pathways in HCC has not been studied.

In our present research, we attempt to explore the precise regulation mechanism of KRT19 on Wnt/Notch signaling pathway in HCC and whether or not there were LncRNAs involving in this regulation. We identified a KRT19 interacting lncRNA Linc-KILH, which specifically binds to KRT19 and restrains its phosphorylation, thus inhibiting the membrane translocation of KRT19 and then enhancing the combining capacity between KRT19 and $\beta$-catenin, which eventually stabilizes and enhances the promoting function of KRT19 on Notch1 pathway in HCC. Moreover, Linc-KILH was overexpressed in HCC tissues, and its deficiency inhibited HCC proliferation and metastasis in vitro and in vivo. As a consequence, Linc-KILH enhanced the malignant phenotypes of hepatoma cells. Our findings further validated the mechanism of LncRNAs which play an oncogenic role through modulating the phosphorylation status of its interacting protein.

\section{Materials and methods}

\section{Patient samples and cell lines}

116 paired pathologically diagnosed HCC samples (including tumor tissues and matched non-tumor liver tissues) were obtained between May 2010 and October 2014 at The Affiliated Changzhou NO.2 People's Hospital of Nanjing Medical University (Changzhou, Jiangsu, China). Written consent approving the usage of tissues in our study were obtained from each patient. The histopathological diagnoses of HCC were conducted by three experienced pathologists independently. This study was approved by the ethnic committee of the Affiliated Changzhou NO.2 People's Hospital of Nanjing Medical University ([2017]KY013-01). Human HCC cell lines (SMMC-7721, Huh7, HepG2, MHCC-97H, MHCC-97L, Hep3B) and the human normal liver L02 cell line were obtained from KeyGen (Nanjing KeyGen Biotech Co., Ltd., Jiangsu, China). All cells used in our study were cultured in Dulbecco's modified Eagle's medium (DMEM, Invitrogen Life Technologies, Carlsbad, CA, USA) supplemented with $10 \%$ fetal bovine serum (FBS, Gibco, Carlsbad, CA, USA) and 1\% streptomycin/ penicillin at $37^{\circ} \mathrm{C}$ in a humidified environment consisting of $95 \%$ air and $5 \%$ CO2.

\section{Real-time quantitative PCR}

To isolate total RNA from cultured cells or tissues, we used TRIZOL reagent (Invitrogen, CA, USA) according to the manufacturer's instructions. Reverse transcription and real-time PCR were then conducted using complementary NDA (cDNA) Synthesis SuperMix (TransGen Biotech, China) and SYBR Green real-time PCR kit (Toyobo, Japan). The primers used in our present study were listed in Table S1. GAPDH served as the internal control.

\section{Ectopic expression and gene silencing}

To effectively knockdown Linc-KILH and KRT19 expression in HCC cells, we designed and constructed corresponding shRNAs and matched negative control shRNAs, and then cloned them into lentivirus vector GV248 (Gene, Shanghai, China), respectively. All the shRNA oligos used in our study are listed in Table S2. In addition, to overexpress Linc-KILH, we subcloned the sequence of Linc-KILH into the lentiviral vector GV367 (Gene, Shanghai, China). All vectors used in our study were labeled with luciferase, and the transfection procedures were manipulated in accordance with the manufacturer's instructions. 


\section{Western blotting and antibodies}

Briefly, protein from cells and tissues were isolated using RIPA lysis buffer and then separated on SDS-PAGE gels and transferred to PVDF membranes. And then, the membranes were blocked with 5\% skim milk in TBST, followed by incubated with the indicated primary antibodies overnight at $4{ }^{\circ} \mathrm{C}$ and then with secondary horseradish peroxidase (HRP)-conjugated secondary antibodies. Antibodies used in our study are as followed: KRT19 (Abcam, ab52625), $\beta$-catenin (Abcam, ab32572), RAC1 (Abcam, ab33186), c-Jun (Abcam, ab32137), Notch1 (Proteintech, 20687-1-AP), Jagged1 (Proteintech, 66890-1-Ig), RBP-Jk (Abcam, ab25949), Hes1 (CST, 11988), CDK2 (Proteintech, 10122-1-AP), CyclinD1 (Proteintech, 60186-1-Ig), Vimentin (Abcam, ab137321), E-cadherin (CST, 3195), GAPDH (Proteintech, 60004-1-Ig). Protein expression signals were detected by a Chemiluminescent Imaging System (Thermo Scientific, IL, USA).

\section{Cell growth, migration and invasion assays}

The growth curve of HCC cells was examined by using Cell Counting Kit-8 (CCK-8) (Dojindo, Japan) and EdU Assay Kit (Guangzhou RIBOBIO, Guangzhou, China) in accordance with the manufacturer's instructions. $5 \times 10^{4}$ cells supplemented with $200 \mu \mathrm{l}$ serum-free DMEM were added into the upper chamber with or without Matrigel (BD Biosciences, San Jose, CA) for invasion or migration invasion assays. 800 $\mathrm{ll}$ DMEM supplemented with $20 \%$ FBS was added into the lower chambers. After incubation at $37{ }^{\circ} \mathrm{C}$ for 24 hours, the cells migrated or invaded to the lower side of the membrane were stained with $0.1 \%$ crystal violet solution, and then imaged and calculated by using a microscope (Olympus, Japan).

\section{In vivo experiments}

To further examine the biological function of Linc-KILH on tumor growth in vivo, tumor subcutaneous xenograft assay was conducted by using six-week-old male nude mice (BALB/c background). A total of $5 \times 10^{6}$ Huh7 and Hep3B cells transfected with indicated vectors were subcutaneously into the left or the right flanks of mice. 5 weeks later, mice were sacrificed and the xenograft were isolated from mice and the volume of each tumor were calculated (length $\times$ width $^{2} \times 0.5$ ). For lung metastasis model, MHCC-97H and Hep3B cells transfected with indicated vectors were suspended in $200 \mu \mathrm{l}$ PBS and then intravenous injected through the tail. 6 weeks later, mice were sacrificed and the lung metastasis situation were detected by the IVIS Lumina II system (Caliper Life
Sciences, MA, USA). The lung of each mice was stained by hematoxylin and eosin and the number of metastasis was calculated. All animal experiments were performed in accordance with the Institutional Animal Care and Use Committee guidelines of the Affiliated Changzhou NO.2 People's Hospital of Nanjing Medical University.

\section{Immunohistochemistry (IHC) assays}

Immunohistochemistry assays were conducted by using paraffin-embedded sections of tissue samples. KRT19 expression was validated in tumor tissues obtained from HCC patients by using anti-KRT19 antibody (Abcam, ab52625). The positive percentage of Ki67 and PCNA was examined in mice xenograft tumor tissues by using anti-Ki67 antibody (Abcam, ab15580) and anti-PCNA antibody (Abcam, ab92552). The tissues were incubated with primary antibodies overnight at $4{ }^{\circ} \mathrm{C}$ and then with HRP-conjugated secondary antibody. Immunohistochemical staining were visualized using DAB. A digitalized microscope camera (Olympus, Japan) was used to visualize and calculate the stained sections.

\section{Subcellular fractionation assays}

To separate the cytoplasm and cytomembrane protein, we used the plasma membrane protein isolation kits (Invent Biotechnologies, Eden Prairie, MN, USA, SM005) in accordance with the manufacturer's instructions. And then, the nuclear fraction was extracted using the nuclear extraction buffer (20 mM HEPES (pH 7.9), $400 \mathrm{mM} \mathrm{NaCl}, 1 \mathrm{mM}$ EDTA, $1 \mathrm{mM}$ EGTA, $1 \mathrm{mM}$ DTT, and $1 \mathrm{mM}$ PMSF) with $10 \mathrm{~min}$ agitation at $4{ }^{\circ} \mathrm{C}$ and subsequently centrifuged at $13,000 \mathrm{rpm}$ for $10 \mathrm{~min}$ at $4^{\circ} \mathrm{C}$.

\section{RNA pulldown assay}

RNA pull-down was performed as previously described [20]. Briefly, biotin-labeled Linc-KILH and control RNA were produced in vitro using Biotin RNA Labeling Mix (Roche) and T7 RNA polymerase (Promega) and then were purified using an RNeasey Mini Kit (Qiagen, Valencia, CA, USA) after treatment with RNase-free DNase I (Roche). Three $\mu g$ biotinylated RNAs were mixed with proteins extracted from Huh7 cells, followed by targeting RNAs with streptavidin beads (Millipore, Bedford, MA, USA). Finally, the retrieved proteins were washed with a RIPA buffer and subjected into SDSPAGE for separation, followed by Western blotting.

\section{RIP and RNA-seq assays}

RIP and RNA-seq assays were performed as previously otherwhere reported [21]. Briefly, to conduct RIP assay, we used the Magna RIPTM RNA Binding Protein Immunoprecipitation Kit (Millipore, 
Massachusetts, USA) in accordance with the manufacturer's instructions. And then, the RNAs gotten from RIP assay were directly applied for the synthesis of ds-cDNA. Next, the ds-cDNA was attached to the adapters and sequenced using Illumina Genome Analyzer (Novogene). Cufflinks was used to quantify the levels of RNAs and then normalized to FPKM. In addition, reads aligned to non-polyA transcripts were excluded. To compute the fold enrichment for RIP, we normalized the FPKM value of each transcript to the average of all transcripts.

\section{Statistical analysis}

All data were analyzed using the appropriate statistical analysis methods with SPSS software (version 19.0, IBM, Armonk, NY), and the data are expressed as the means \pm SD. Student's t-test was used to analyze the differences between 2 groups, while one-way ANOVA was applied for multiple comparisons. Overall survival (OS) and recurrencefree survival (RFS) were assessed using Kaplan-Meier method and the significance was determined by log-rank test. $P$-value $<0.05$ was considered as
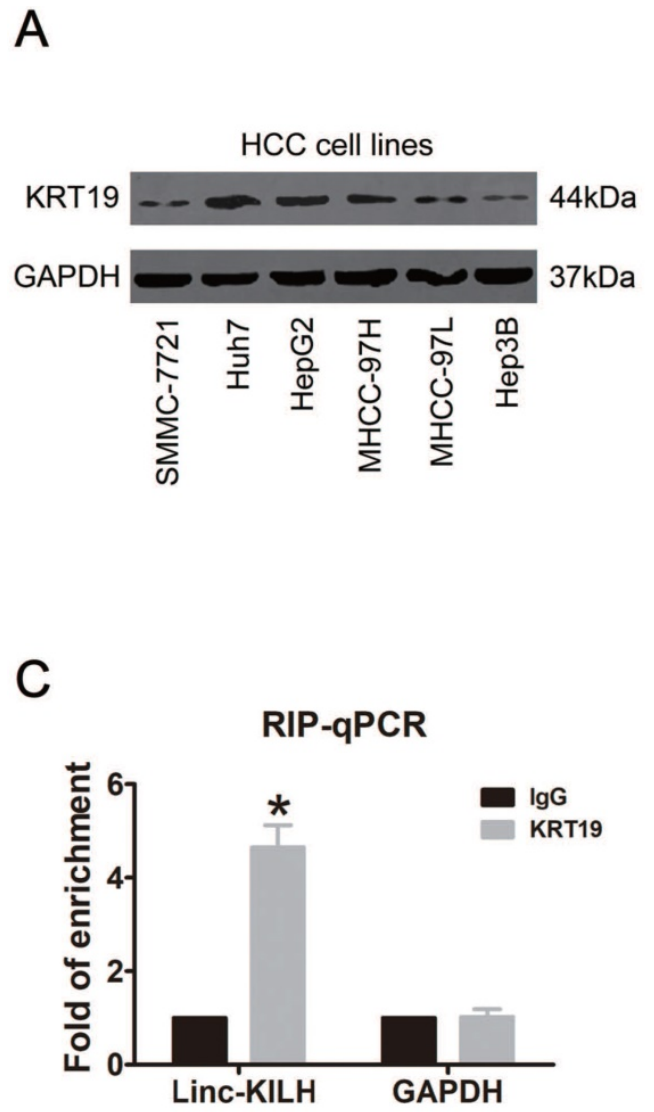

Figure 1. KRT19 binds a lincRNA Linc-KILH in HCC cells. (A) The expression level of KRT19 in HCC cell lines was detected by immunoblotting. (B) Huh7 cells were subjected to RIP assays with a KRT19 antibody or isotype-matched control IgG, RIP-Seq analysis showing average-normalized, log2 transformed FPKM value of KRT19-RIP and input. (C) The amount of Linc-KILH in the precipitates was analyzed by quantitative real-time PCR analysis. RNA enrichment indicates the RNA levels of Linc-KILH or GAPDH in the anti-KRT19 precipitates relative to those in the IgG precipitates. D, Biotin-labeled Linc-KILH and Linc-KILH-AS were incubated with Huh7 cell lysate, and KRT19 interaction was confirmed by Western blotting. $* P<0.05$.

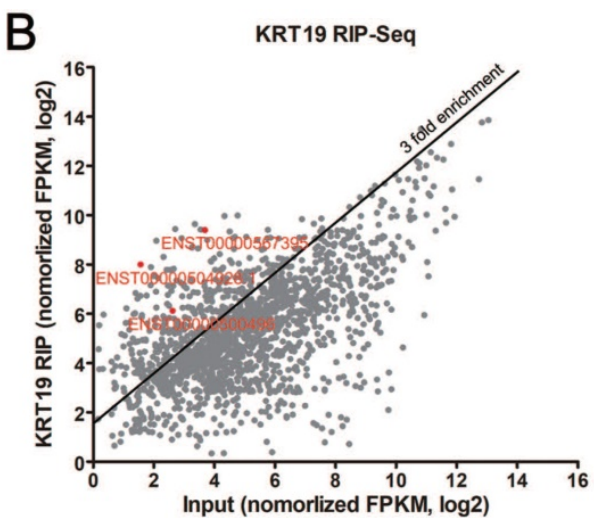

statistical significance.

\section{Results}

\section{KRT19 binds a lincRNA Linc-KILH in HCC cells}

KRT19 was reported to express in various cancers, including HCC, but $10 \%-28 \%$ of hepatocellular carcinomas were reported to express KRT19, and those HCC patients with KRT19 positive were found to display a more invasive phenotype and poorer prognosis $[5,22,23]$. Here, we checked the protein level of KRT19 in 116 HCC tissue samples by immunohistochemistry staining (IHC), and KRT19 positive expression was found in 26 cases $(26 / 116$, $22.4 \%$ ) (Figure S1A). In addition, we examined the mRNA level of KRT19 in the HCC tissue mentioned above by RT-qPCR (Figure S1B). Also, KRT19 protein level was evaluated in HCC cell lines (Figure 1A). Similarly, we got a differential expression pattern of KRT19 in HCC cells and Huh7 cells showed the highest expression.

The differential expression of KRT19 in HCC tissues and cell lines and its function in mediating

B

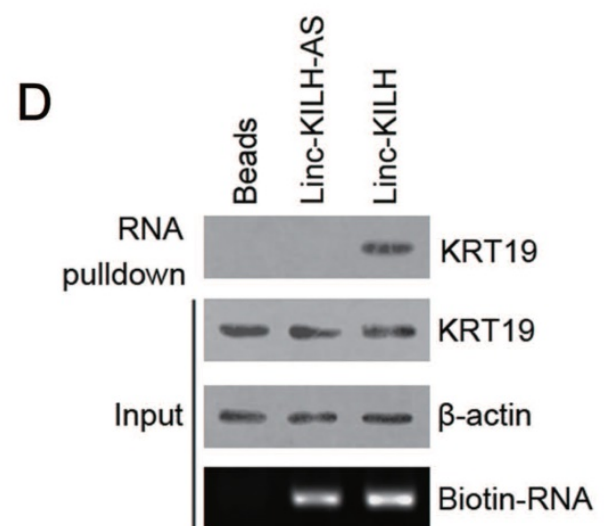


Notch1 signaling indicates that there may exist other regulators which could regulate its signal adjusting function [5-7]. Here, we proposed to identify KRT19 interacting RNAs, especially long noncoding RNAs. RNA immunoprecipitation and sequencing (RIP-Seq) was conducted in KRT19 highest expression Huh7 cells. Using > 3-fold enrichment and FPKM value > 2 in input as a cutoff, we identified a total of 256 transcripts bound by KRT19, excitingly, three LncRNAs were found bound by KRT19. In addition, we found that ENST00000504928.1 showed the richest content in IP sample compared with input (Fold of enrichment $\sim 80$ ) (Figure 1B) (Table S3). Next, we examined the levels of these 3 LncRNAs in 20 pairs of randomly selected, paired tumor and corresponding non-tumor liver tissues by RT-qPCR, and found that only ENST00000504928.1 was remarkably overexpressed in HCC tumor tissues compared with the corresponding non-tumor liver tissues (Figure S2). Therefore, we named it as KRT19 interacting long noncoding RNA in hepatocellular carcinoma (Linc-KILH). Next, we confirmed the binding association between KRT19 and Linc-KILH by RIP-qRCR, RNA pulldown and Western blotting analysis (Figure 1C and 1D).

\section{Linc-KILH expression is upregulated in HCC tissues and correlated with prognosis of patients with HCC}

To further validate the increase of Linc-KILH, its expression was further analyzed by RT-qPCR in 116 pairs of HCC tumor and non-tumor tissues. We revealed that Linc-KLIH was obviously overexpressed in tumorous tissues compared with the corresponding non-tumor liver tissues (Figure 2A). In addition, we found that the expression of Linc-KILH in KRT19 positive HCC tissues was significantly higher than that in KRT19 negative HCC tissues (Figure S1C). Next, we investigated the expression pattern of Linc-KILH in HCC cells, and validated that Linc-KILH was also up-regulated in most HCC cell lines compared with normal liver cell L02 (Figure 2B). And then, its coding potential was examined by using Coding Potential Calculator and CPAT [24, 25], and results from both program indicated that Linc-KILH has no coding ability (Figure S3A and S3B). Intracellular location of Linc-KILH was visualized in Huh7 and MHCC-97H cells by RNA fluorescence in situ hybridization (FISH) assays, and it mainly locates in the cytoplasm of HCC cells (Figure 2C). To assess whether the aberrant expression of Linc-KILH and KRT19 are related to HCC progression, the clinical information was collected and analyzed in HCC patients mentioned above. As shown in Table 1, we divided all 116 patients into Linc-KILH high and low groups according to the median value of Linc-KILH, and KRT19 positive and negative groups according to the results of KRT19 IHC staining. Statistical analysis shown that both high Linc-KLIH and positive KRT19 expression was obviously associated with increased tumor size $(P=0.001$ for Linc-KILH; $P=0.001$ for KRT19), severer microvascular invasion $(P=0.009$ for Linc-KILH; $P=0.008$ for KRT19), and more intrahepatic metastasis $(P=0.010$ for Linc-KILH; $P=$
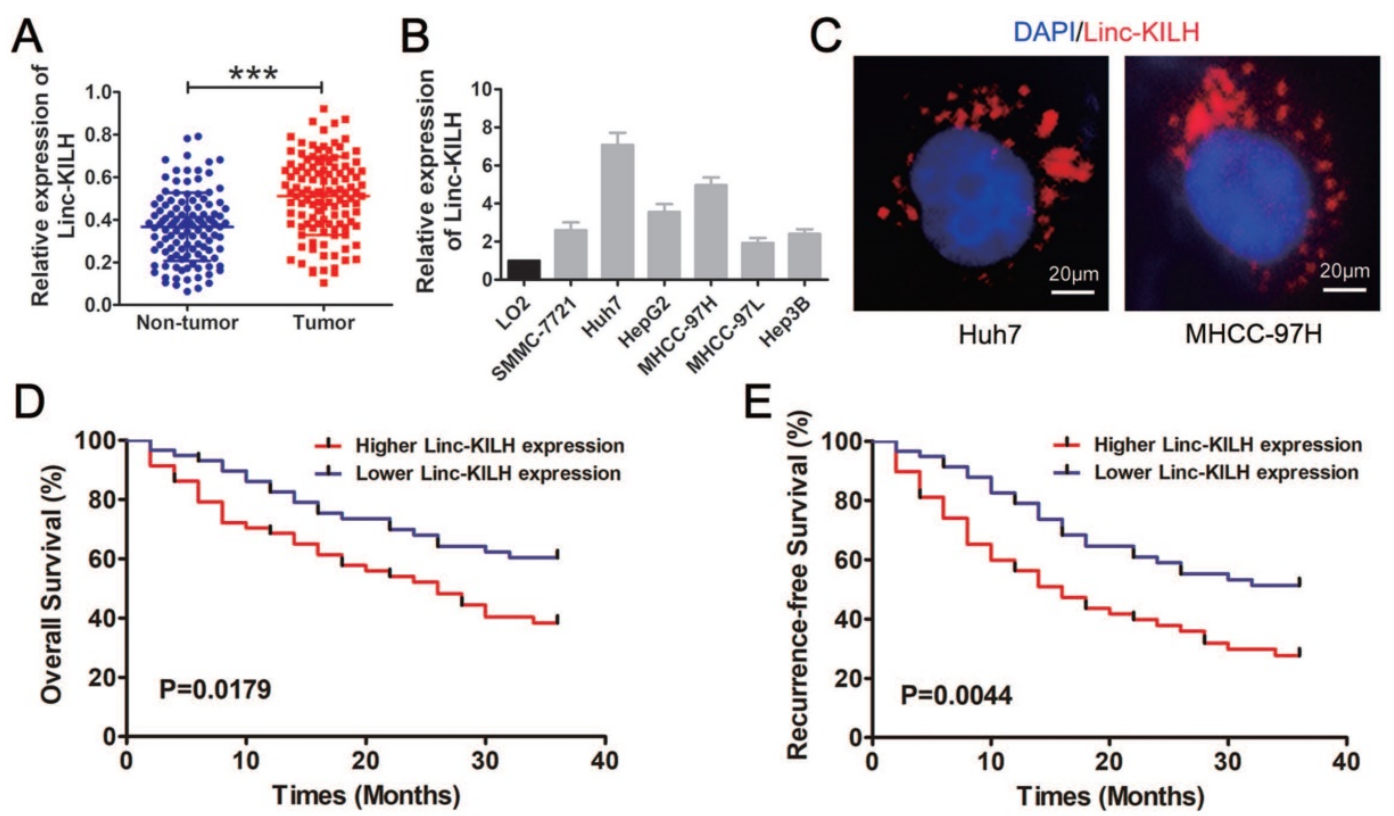

Figure 2. Linc-KILH is up-regulated in HCC tissues and correlated with the prognosis of patients with HCC. (A) The expression level of Linc-KILH was detected by RT-qPCR in HCC tumor tissues and adjacent non-tumor liver tissues. (B) Relative expression of Linc-KILH in human L02 hepatocytes and HCC cell lines was detected by RT-qPCR. (C) The subcellular location of Linc-KILH was analyzed through fluorescence in situ hybridization (FISH) (original magnification $\times 400)$. (D) The overall survival and recurrence-free survival rates of $116 \mathrm{HCC}$ patients were compared between higher Linc-KILH and lower Linc-KILH groups. $* * * P<0.001$. 
0.025 for KRT19). Furthermore, Kaplan-Meier and log-rank test analyses revealed that higher Linc-KILH expression was obviously associated with decreased overall survival (OS) and recurrence-free survival (RFS) rates $(P=0.0179$ for OS and $P=0.0044$ for RFS) (Figure 2D and 2E). In addition, KRT19 positive expression in HCC tissues obviously correlated with decreased overall survival (OS) and recurrence-free survival (RFS) rates $(\mathrm{P}=0.0363$ for $\mathrm{OS}$ and $\mathrm{P}=0.0027$ for RFS) (Figure S1D and S1E).

Table 1. Correlation between Linc-KILH and KRT19 expression and clinicopathological characteristics of HCC patients

\begin{tabular}{|c|c|c|c|c|c|c|}
\hline \multirow[t]{2}{*}{ Characteristics } & \multicolumn{2}{|c|}{ Linc-KILH } & \multirow[t]{2}{*}{$P$ value } & \multicolumn{2}{|l|}{ KRT19 } & \multirow[t]{2}{*}{$P$ value } \\
\hline & High & Low & & Positive & Negative & \\
\hline All cases & 58 & 58 & & 26 & 90 & \\
\hline Age, years & & & 0.850 & & & 0.833 \\
\hline$<60$ & 35 & 34 & & 15 & 54 & \\
\hline$\geq 60$ & 23 & 24 & & 11 & 36 & \\
\hline Gender & & & 0.342 & & & 0.590 \\
\hline Male & 49 & 45 & & 22 & 72 & \\
\hline Female & 9 & 13 & & 4 & 18 & \\
\hline AFP & & & 0.678 & & & 0.554 \\
\hline$\leq 20 \mathrm{U} / \mathrm{L}$ & 17 & 15 & & 6 & 26 & \\
\hline$>20 \mathrm{U} / \mathrm{L}$ & 41 & 43 & & 20 & 64 & \\
\hline HBsAg & & & 0.226 & & & 0.304 \\
\hline Positive & 50 & 45 & & 23 & 72 & \\
\hline Negative & 8 & 13 & & 3 & 18 & \\
\hline Liver cirrhosis & & & 0.696 & & & 0.155 \\
\hline Yes & 39 & 37 & & 20 & 56 & \\
\hline No & 19 & 21 & & 6 & 34 & \\
\hline Tumor size & & & 0.001 & & & 0.001 \\
\hline$\leq 5 \mathrm{~cm}$ & 19 & 36 & & 5 & 50 & \\
\hline$>5 \mathrm{~cm}$ & 39 & 22 & & 21 & 40 & \\
\hline Tumor number & & & 0.243 & & & 0.316 \\
\hline Single & 44 & 49 & & 19 & 74 & \\
\hline Multiple & 14 & 9 & & 7 & 16 & \\
\hline Microvascular invasion & & & 0.009 & & & 0.008 \\
\hline Yes & 25 & 12 & & 14 & 23 & \\
\hline No & 33 & 46 & & 12 & 67 & \\
\hline Intrahepatic metastasis & & & 0.010 & & & 0.025 \\
\hline Yes & 12 & 3 & & 7 & 8 & \\
\hline No & 46 & 55 & & 19 & 82 & \\
\hline Edmondson & & & 0.342 & & & 0.590 \\
\hline I-II & 9 & 13 & & 4 & 18 & \\
\hline III-IV & 49 & 45 & & 22 & 72 & \\
\hline
\end{tabular}

Total data from 116 HCC patients were analyzed. For the expression of Linc-KILH, median expression level was used as the cutoff. Data were analyzed by chi-squared test. $P$-value in bold indicates statistically significant.

\section{Linc-KILH enhances the proliferation, migration and invasion abilities of KRT19 positive HCC cells in vitro}

According to the expression level of Linc-KLIH and KRT19 in HCC cell lines, we defined Huh7 and MHCC-97H as KRT19-positive cells, Hep3B as KRT19-negative cells to further explore whether or not Linc-KILH participate in the physiology function of KRT19 in HCC cells. Three independent shRNA plasmids were generated and transfected in Huh7, MHCC-97H and Hep3B cells, as shown in Figure 3A, only shRNA-1 exhibited the most knockdown efficiency and it was applied to investigate the biological function of Linc-KILH on HCC cells. CCK8 assay was used to determine the proliferation ability of HCC cells, knockdown of Linc-KILH prominently suppressed the proliferation of Huh7 and MHCC-97H cells, whereas, no change was gotten in Hep3B cells (Figure 3B). In addition, EdU (5-ethynyl-2'-deoxyuridine) assay was conducted and the same results were gotten (Figure 3C). In addition, we conducted transwell assays to investigate HCC cells migration and invasion abilities and found that silence of Linc-KILH obviously inhibited the migration and invasion abilities in KRT19-positive Huh7 and MHCC-97H cells while no significant change was obtained in KRT19-negative Hep3B cells (Figure 4A and 4B). Based on the functional experiments above, we confirmed that Linc-KILH significantly enhanced the proliferation, migration and invasion abilities of KRT19 positive HCC cells in vitro.

\section{Linc-KILH facilitates KRT19 positive HCC cells growth and metastasis in vivo}

We then determined the biological function of Linc-KILH on HCC growth and metastasis in vivo. Here, mouse subcutaneous xenograft models were established, we injected Huh7 and Hep3B cells with stable silencing of Linc-KILH subcutaneously in nude mice. Tumor xenografts derived from Linc-KILH silencing Huh7 cells had smaller mean volumes compared with control cells, while no difference was gotten in Hep3B cells (Figure 5A). Next, we detected the positive rates of Ki67 and PCNA in the tumor tissues from the subcutaneous xenograft models by immunohistochemistry. As shown in Figure 5B, Linc-KILH silencing decreased the positive percentage of Ki-67 and PCNA staining cells in Huh7 cells, whereas no alteration was gained in Hep3B cells. To further explore the biological function of Linc-KILH on HCC metastasis in vivo, we conducted mice lung metastasis models by tail vein injecting Linc-KILH stably silenced MHCC-97H and Hep3B cells which were labeled with firefly luciferase. After injection for 6 weeks, mice were sacrificed and metastases were evaluated. Obviously, the bioluminescent signals were significantly reduced in the Linc-KILH knockdown MHCC-97H cells, while no significant change was observed in Hep3B cells (Figure 5C). H\&E staining further confirmed a reduction of metastatic lesions in the lung of mice that received Linc-KILH-knockdown MHCC-97 cells (Figure 5D). In summary, our present data suggest that Linc-KILH enhances the growth and metastasis abilities of KRT19 positive HCC in vivo. 

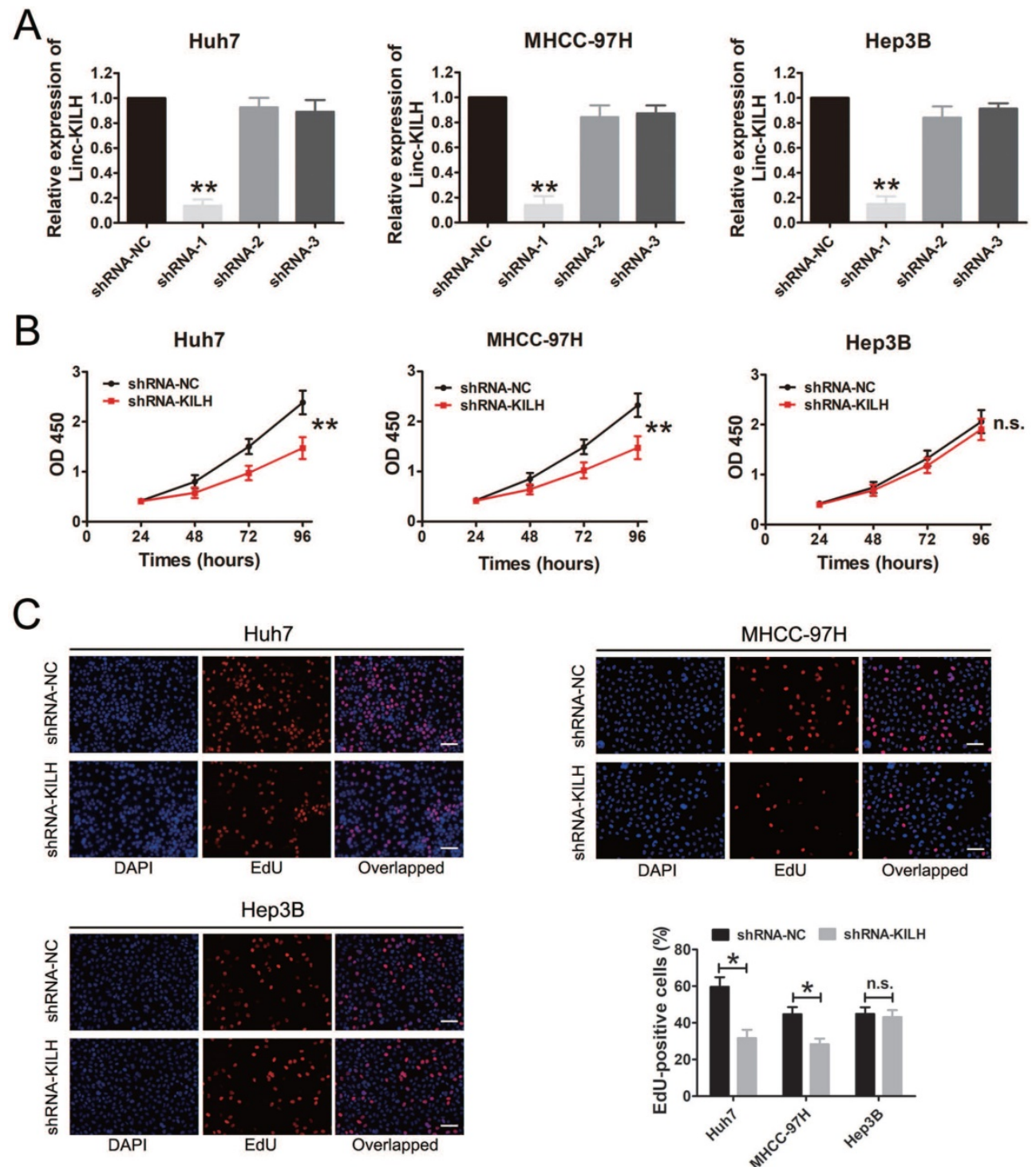

Figure 3. Linc-KILH promotes HCC cell proliferation in vitro. (A) Gene silence of Linc-KILH using Linc-KILH shRNAs in Huh7, MHCC-97H and Hep3B cells was conducted and the transfection efficiency was validated by RT-qPCR. (B) Cell proliferation assays for Huh7, MHCC-97H and Hep3B cells infected with the lentivirus silencing Linc-KILH or the control using Cell Counting Kit-8 assay. (C) EdU staining was used to detected the proliferation abilities of HCC cells knockdown of Linc-KILH (original magnification $\times 100$ ). Each experiment was performed in triplicate and the results are shown as the means \pm SD. $* P<0.05 ; * * P<0.01$.

\section{Linc-KILH inhibits the phosphorylation of KRT19 to potentiate Notch 1 signaling in HCC}

Studies have revealed that one of the most prevalent mechanisms underlying the interaction between LncRNAs and their binding proteins was adjusting the phosphorylation status of proteins [19, 26, 27]. The phosphorylation status of KRT19 on Ser35 has been recognized as the most important regulation pattern of KRT19 in various biological behaviors, including tumors [28-30]. Since Linc-KILH was identified bound to KRT19 and could influence the biological properties of HCC cells with the existence of KRT19, we proposed that it might participate in the regulation of the phosphorylation of KRT19. To confirm this hypothesis, we detected the protein content of the phosphorylated KRT19 on Ser35 and the total KRT19 by immunoblotting in HCC cells. As shown in Figure 6A, silence of Linc-KILH in KRT19-positive Huh7 and MHCC-97H cells obviously increased the phosphorylation status but not the total content of KRT19, whereas no alteration was gained in KRT19-negative Hep3B cells. Studies have reported that once KRT19 was phosphorylated on Ser35, it would remodel its form from filamentous to granulous mold and translocate from the cytoplasm to cell membrane. Since Linc-KILH could adjust KRT19 phosphorylation, we suspect that it might promote the translocation of KRT19 in HCC cells. We extracted and isolated proteins from cell membrane and cytoplasm with a commercial kit and examined the protein content of the phosphorylated and total KRT19 respectively. As exhibited in Figure 6B, KRT19 phosphorylated on Ser35 mainly located in the cell 
membrane and knockdown of Linc-KILH strengthened the translocation of KRT19 from cytoplasm to cell membrane in Huh7 and MHCC-97H cells.

Studies investigating the regulation function of KRT19 on Wnt/Notch signaling in tumors have gained an opposing result, and the key point underlying this opposing regulation was whether KRT19 could bind to RAC1 in cytoplasm of tumor cells $[6,7]$. In mammary cancer, KRT19 was reported to interact with $\beta$-catenin/RAC1 complex and then upregulate NUMB expression, thus suppressing Notch signaling [6]. However, in colon cancer, KRT19 was validated to interact with $\beta$-catenin but not with RAC1 and then enhance the transcription function of LEF/TCF, thus promoting Notch signaling [7]. Now that researches have validated the promoting function of KRT19 on Notch1 signaling in HCC cells, we proposed that the function of KRT19 in HCC might be
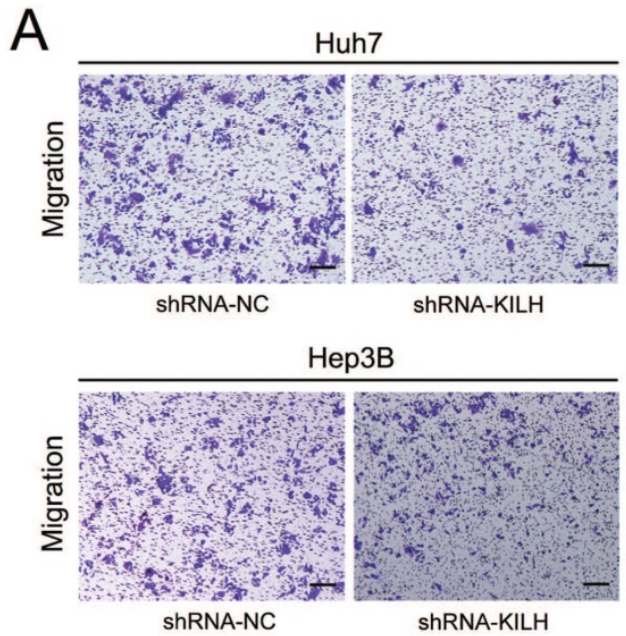

B
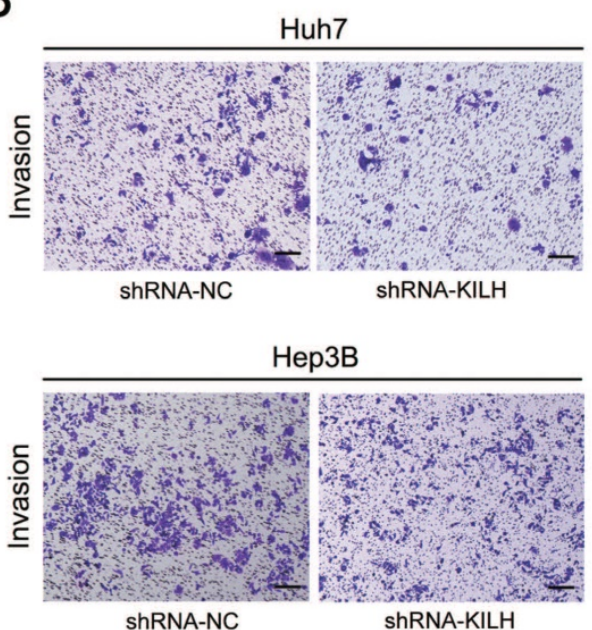

similar with that in colon cancer [5, 31]. Co-IP assays was conducted to explore this hypothesis, and the results proved that KRT19 only interacted with $\beta$-catenin, but not RAC1 in HCC cells (Figure 6C). The interaction of KRT19 and $\beta$-catenin in cytoplasm stabilized and enhanced the translocation of $\beta$-catenin from cytoplasm to nucleus and then enhanced the Notch signaling. Here, we validated that Linc-KILH could inhibited the phosphorylation of KRT19 and strengthened its cytoplasmic expression. Next, we aimed to examine the function of Linc-KILH on the translocation of $\beta$-catenin in HCC cells. We found that knockdown of Linc-KILH in Huh7 and MHCC-97H cells attenuated nuclear translocation of $\beta$-catenin, while there was no change of the nuclear RAC1 content upon Linc-KILH silence (Figure 6D). Therefore, we elucidated that KRT19 interacted with $\beta$-catenin and Linc-KILH could regulated $\beta$-catenin nuclear import but not RAC1 in HCC cells.
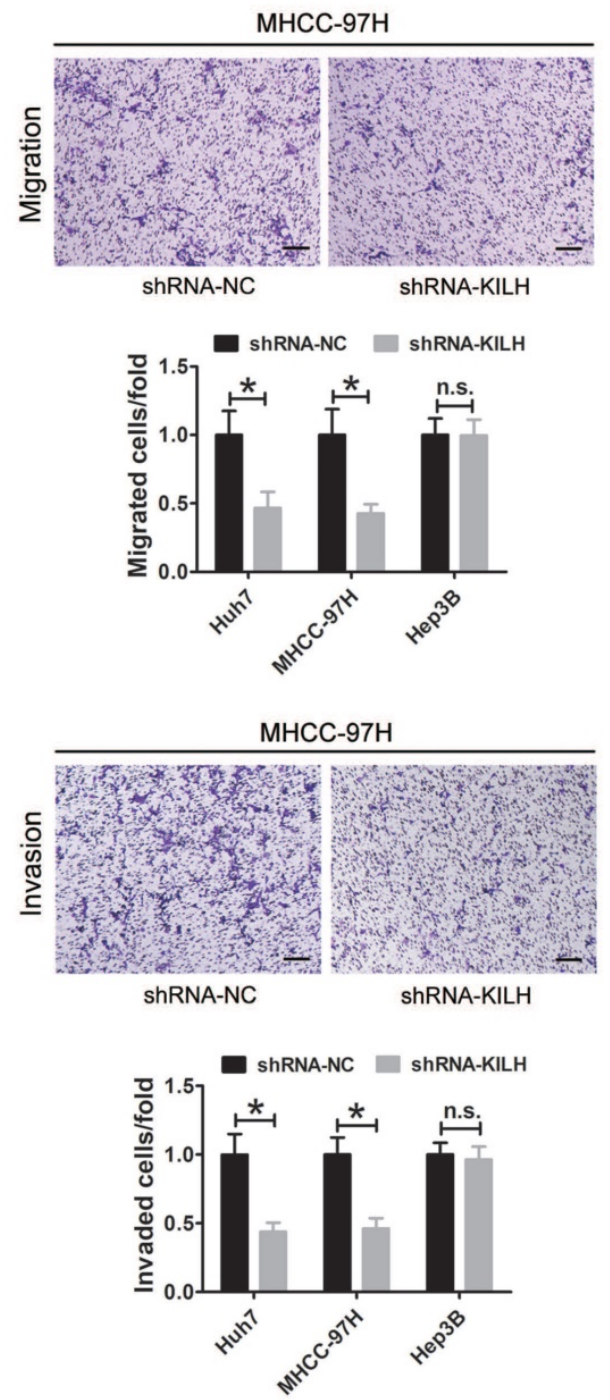

Figure 4. Linc-KILH enhances the migration and invasion abilities of HCC cells in vitro. (A and B) Transwell assays were conducted to examined the migration and invasion abilities of Huh7, MHCC-97H and Hep3B cells transfected with Linc-KILH shRNA (original magnification $\times 100$ ). Each experiment was performed in triplicate and the results are shown as the means \pm SD. $* P<0.05$. 
A

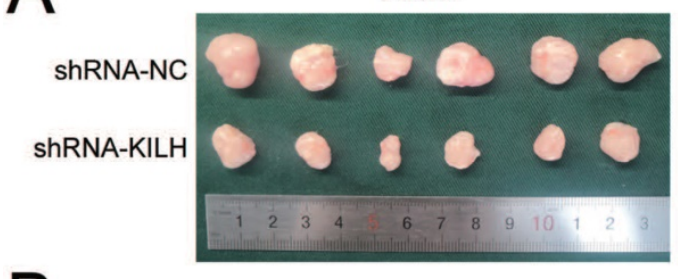

D
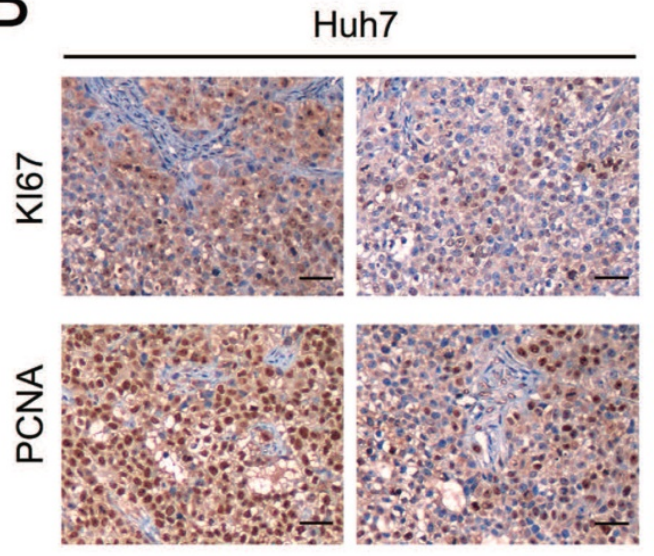

shRNA-NC

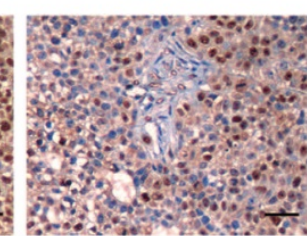

ShRNA-KILH

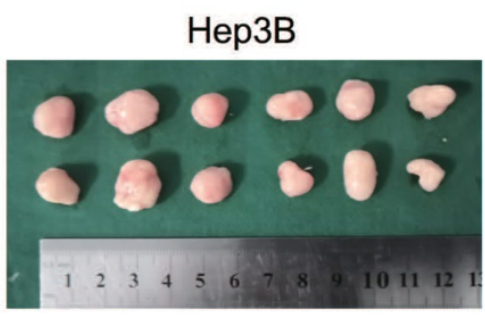

Нер3B
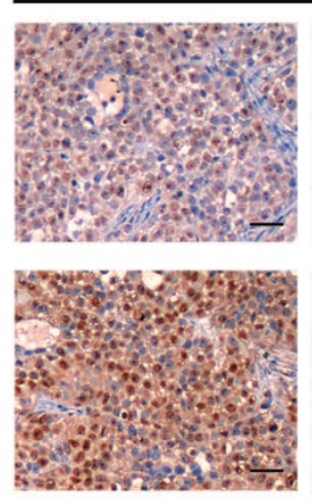

ShRNA-NC

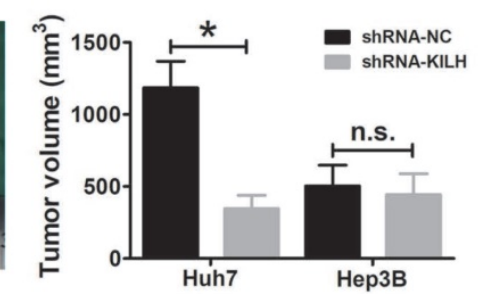

Hep3B
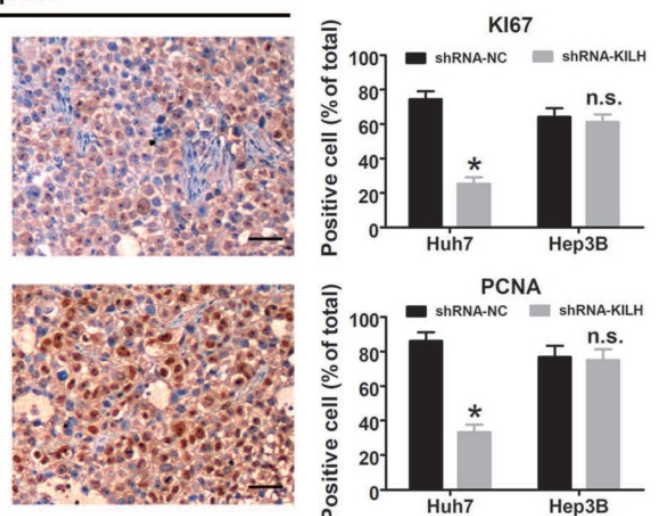

ShRNA-KILH

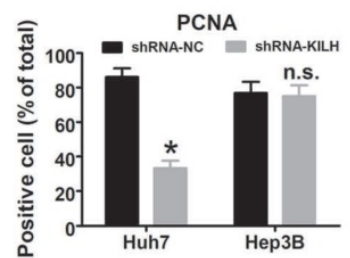

C
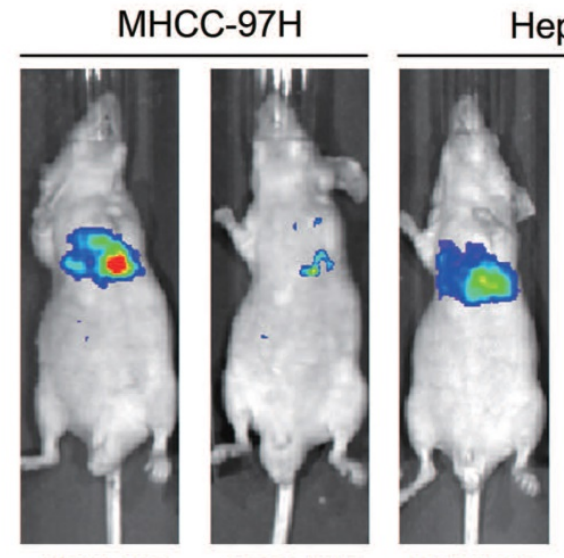

Нер3B
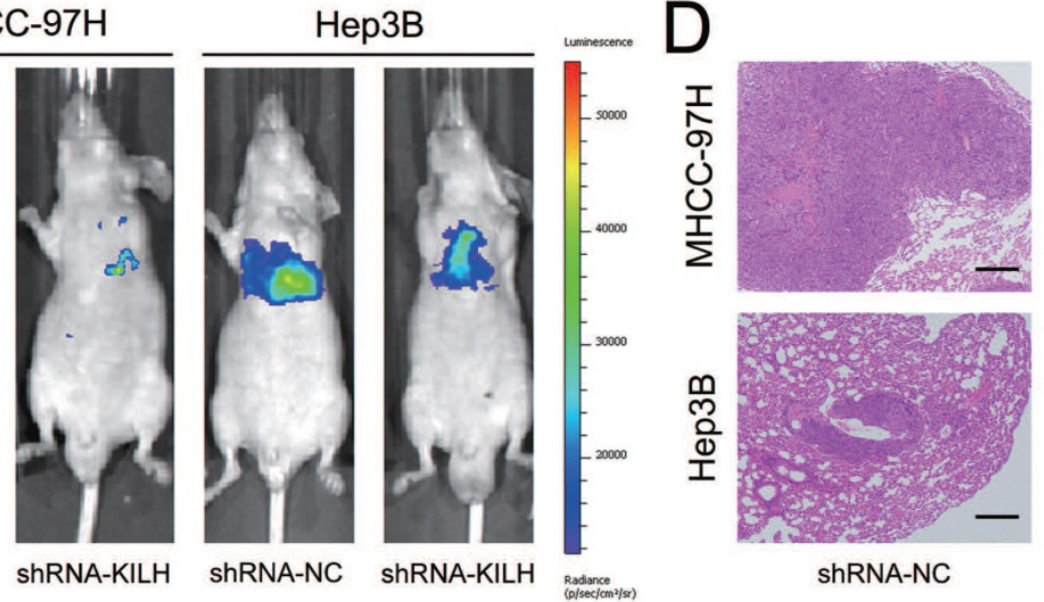

ShRNA-NC
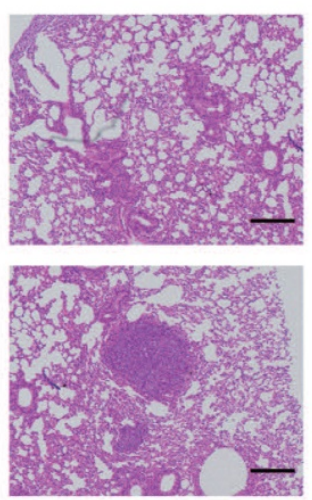

shRNA-KILH

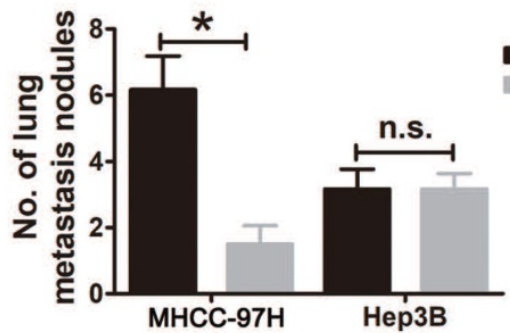

Figure 5. Linc-KILH facilitates HCC growth and metastasis in vivo. (A) Huh7 and Hep3B cells knockdown of Linc-KILH and the control cells were subcutaneously injected into 6-week-old BALB/c nude mice, 5 weeks later, the mice were sacrificed and the tumor size was measured. (B) The percentage of Ki-67 and PCNA positive staining cells was detected by immunohistochemistry in the tumor tissues from the subcutaneous xenograft model (original magnification $\times 100$ ). (C) A lung metastasis model was established in which mice were injected with HCC cells $\left(5 \times 10^{6}\right.$ cells suspended in $200 \mu \mathrm{L}$ PBS $)$ through the tail vein and the lung metastasis was investigated respectively using the IVIS Lumina II system. Representative images of a mouse in each group were presented. (D) All the results of lung colonization were validated by the histological examination (H\&E) (original magnification $\times 100$ ). Metastatic tumors with volumes $>2 \mathrm{~mm}^{3}$ were identified and compared in each group. Experiments were performed in triplicate independently and the results are shown as the means $\pm \mathrm{SD}$. $* P<0.05$.

Next, we confirmed the regulation effect of Linc-KILH on Notch1 signaling in HCC cells by immunoblotting. We found that silence of Linc-KILH suppressed Notch1 signaling in KRT19-positive Huh7 and MHCC-97H ells, and no difference was observed in KRT19-negative cells (Figure 6E). Notch1 signaling has been reported as a cancer promoting pathway in HCC through enhancing tumor cell proliferation, 
migration, invasion and EMT transformation. Here, we detected some related markers (CDK2, CyclinD1, Vimentin, E-cadherin) by immunoblotting and found that knockdown of Linc-KILH in Huh7 and MHCC-97H cells reduced the protein levels of CDK2, CyclinD1 and Vimentin, but increased E-cadherin protein level, while no change was obtained in Hep3B cells (Figure 6F). Taken together, we validated that Linc-KILH could inhibit the phosphorylation of KRT19 on Ser35 and then enhanced its translocation from cytoplasm to membrane thus inhibiting $\beta$-catenin nuclear import, and finally potentiated Notch1 signaling in HCC.

\section{KRT19 is essential for the tumor promoting function of Linc-KILH in HCC}

In vitro and in vivo functional experiments in Huh7, MHCC-97H and Hep3B cells revealed that the cancer promoting function of Linc-KILH on HCC cells was dependent on KRT19 expression. To further validate the necessity of KRT19 for Linc-KILH, we conducted a series of rescue experiments. Firstly, we overexpressed Linc-KILH in Huh7 cells and the transfection efficiency was examined by RT-qPCR (Figure S4A). And then, we silenced KRT19 in Linc-KILH overexpressed Huh7 cells, and the knockdown efficiency was examined by RT-qPCR and immunoblotting respectively (Fig. S4B and S4C). EdU assays was conducted to examine the proliferation ability of HCC cells, overexpression of Linc-KILH significantly enhanced the growth of Huh7 cells, whereas knockdown of KRT19 practically abolished the enhancement effect of Linc-KILH (Figure 7A). Similarly, transwell assays demonstrated that silence of KRT19 almost eliminated the promoting function of Linc-KILH on the migration and invasion abilities of
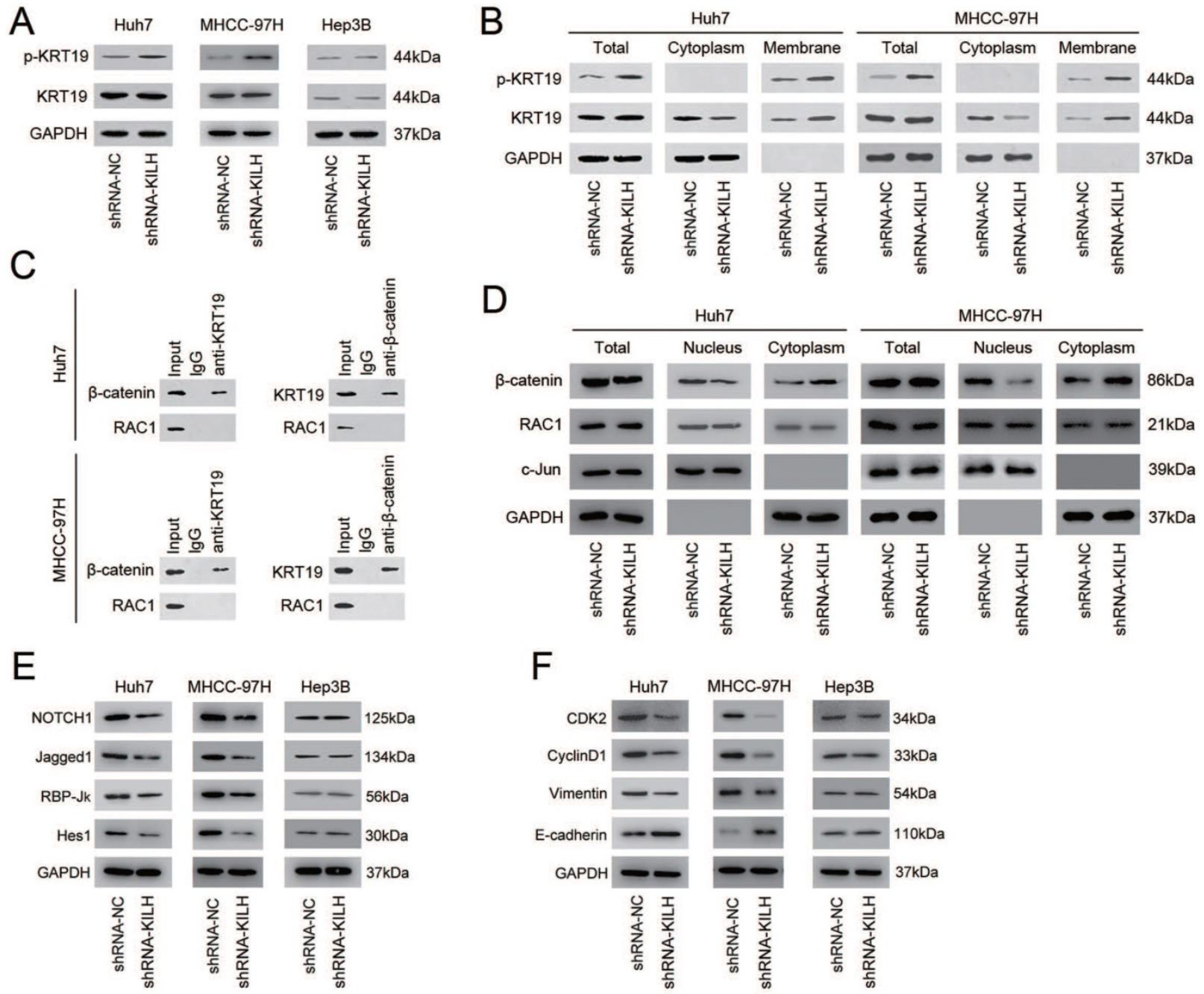

Figure 6. Linc-KILH inhibits the phosphorylation of KRT19 to potentiate Notchl signaling in HCC. (A) The expression levels of total and phosphorylated KRT19 on Ser35 site in Huh7, MHCC-97H and Hep3B cells upon Linc-KILH alteration were examined by western blotting. (B) The subcellular location and the content of the phosphorylated and total KRT19 were detected by western blotting in KRT19 positive Huh7 and MHCC-97H cells. (C) Co-immunoprecipitation (Co-IP) was performed using Protein A/G Sepharose and antibodies specific for KRT19, $\beta$-catenin, and RACl, or normal IgG in KRT19 positive Huh7 and MHCC-97H cells. Cell lysates were analyzed by Western blotting. (D) Cell fractionation assay for $\beta$-catenin and RACl analyzed by Western blotting; actin and c-Jun were used as the cytoplasmic and nuclear markers, respectively, in KRT19 positive Huh7 and MHCC-97H cells. (E) The variation of the expression of the members of Nothcl signaling pathway in Linc-KILH-silenced cells was determined by western blotting. (F) The expression levels of cell proliferation and EMT transformation related genes were examined by western blotting in HCC cells upon Linc-KILH silence. 
HCC cells (Figure 7B). As shown in Figure 7C, the in vivo subcutaneous experiments certified that overexpression of Linc-KILH obviously strengthened the growth capacity of Huh7 cells, and knockdown of KRT19 removed the effect of Linc-KILH. Results from the mice metastasis model also confirmed this phenomenon (Figure 7D). Collectively, these data further demonstrated that the cancer promoting function of Linc-KILH was dependent on the presence of KRT19 in HCC cells.

\section{Discussion}

As far as we know, this is the first discovery of long noncoding RNA bound to KRT19 (Linc-KILH) and explores its biological function in HCC. We demonstrated Linc-KILH was significantly upregulated in HCC tissues compared with the corresponding non-tumor tissues in our study cohort. Correlation analysis revealed that high Linc-KILH expression was positively correlated with advanced tumor progression and poorer prognosis of HCC patients. Functionally, silence of Linc-KILH significantly repressed the growth and metastasis abilities of KRT19 positive HCC cells in vitro and in vivo. Therefore, our study suggested that Linc-KILH could function as a crucial factor for the regulation of HCC progression.

With a large number of LncRNAs been fully explored, many aspects of their biological and physiological functions and the underlying mechanisms have been validated [13, 15, 32, 33]. One of the most prevalent functional patterns of LncRNAs is forming complexes with proteins and then regulation the status of the binding proteins through
A
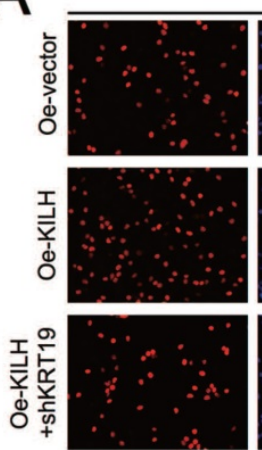

EdU
Huh7
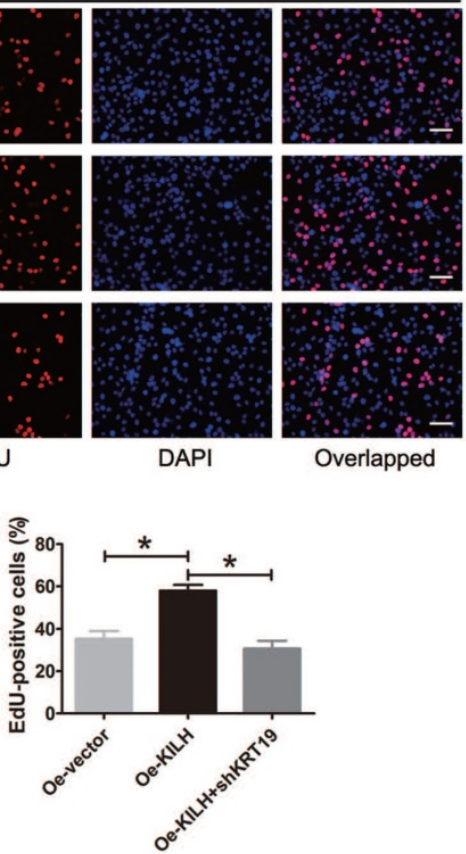

C

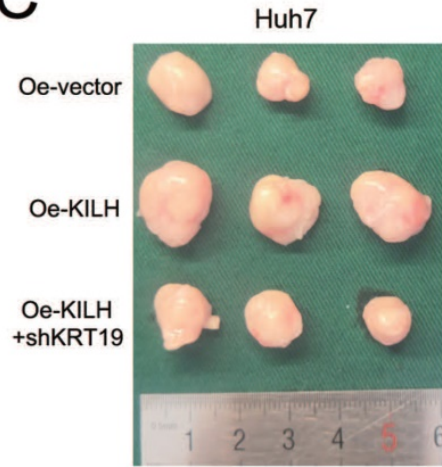

$\mathrm{B}$

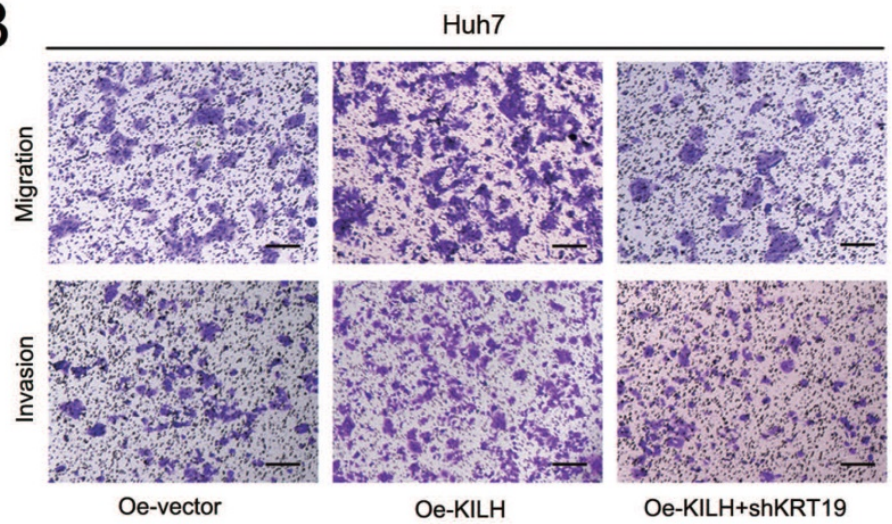

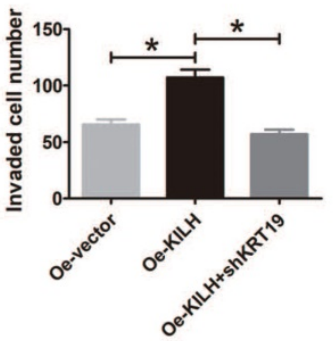

D
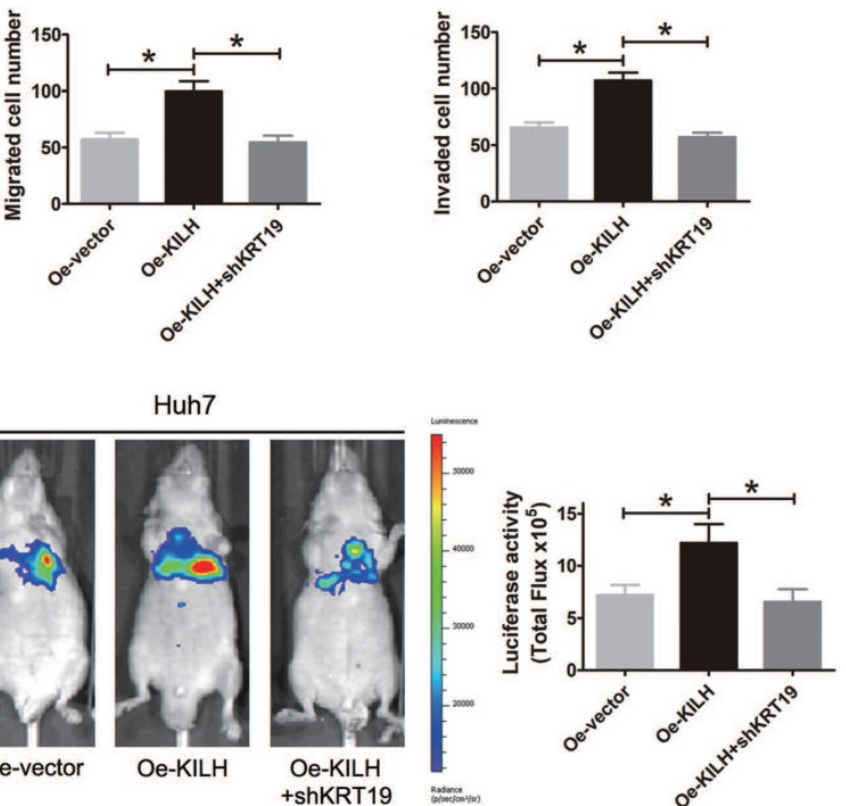

Figure 7. KRT19 is essential for the tumor promoting function of Linc-KILH in HCC. (A) EdU staining was used to detected the proliferation abilities of HCC cells when Linc-KILH was overexpressed with and without KRT19 silence in Huh7 cells (original magnification $\times 100$ ). (B) Transwell assays were conducted to examined the migration and invasion abilities of Linc-KILH overexpressed Huh7 cells upon KRT19 silence (original magnification $\times 100)$ ). (C) Images and volume of xenografts established by subcutaneous transplantation with Linc-KILH overexpression and KRT19 knockdown were exhibited and calculated. (D) Representative images of the lung metastasis model and the luciferase activities were measured by IVIS imaging system. Each experiment was performed in triplicate and the results are shown as the means \pm SD. $* P<0.05$. 
various posttranslational modifications [34-38]. For instance, TSLNC8 could function as a tumor suppressor through physically interacting with STAT3 and modulating its phosphorylation on Tyr705 and Ser727 sites [38]. Lnc-EGFR was reported to specifically bind to EGFR and then restrained its ubiquitination, thus stimulating Treg cells differentiation in HCC [34]. KRT19 has been demonstrated as a tumor promoting factor in various tumors, including HCC, whereas the detailed underlying mechanisms are still sciolistic [5-7]. In our present study, we proposed to concentrate on KRT19 interacting RNAs, especially LncRNAs. We conducted RIP-Seq analysis in KRT19 highest expression Huh7 cells and successfully identified a long noncoding RNA Linc-KILH interacting with KRT19 in HCC. Followed RIP-qPCR, RNA pulldown and immunoblotting assays reconfirmed the physically interacting between Linc-KILH and KRT19 in HCC cells. Furthermore, we demonstrated that Linc-KILH could inhibit the phosphorylation status of KRT19 on Ser35 site. Studies have revealed that once KRT19 was phosphorylated on Ser35, it would transform from filamentous form to granulous form and translocate from cytoplasm to cell membrane. Here, we demonstrated that Linc-KILH participated in this translocation regulation and promoted the membrane translocation of KRT19.

An opposing concept has been acquired on the regulation function of KRT19 on Wnt/Notch signaling in tumors [7]. KRT19 could interact with $\beta$-catenin/RAC1 complex and then upregulate NUMB expression, thus suppressing Notch signaling in mammary tumor [6]. However, in colon cancer, KRT19 was reported to interact with $\beta$-catenin, but not RAC1, and then enhanced the transcription function of LEF/TCF, thus promoting Notch signaling [7]. The critical factor on the basis of this opposing regulation is whether KRT19 could bind to RAC1 in the cytoplasm of tumor cells. However, KRT19 was validated to promote Nothc1 signaling in HCC, thus, these discoveries encouraged us to explore the detailed roles of KRT19 on Notch1 signaling in HCC. Here, we present strong evidence that KRT19 interact with $\beta$-catenin but not RAC1 in HCC cells. Linc-KILH enhanced the interaction of $\beta$-catenin of KRT19 in cytoplasm and promoted the nuclear translocation of $\beta$-catenin in HCC cells. Signaling detecting revealed that Linc-KILH could enhance the promoting function of KRT19 on Notch1 signaling with the existence of KRT19 in HCC cells. In vitro and in vivo functional experiments in Huh7, MHCC-97H and Hep3B cells revealed that the cancer promoting function of Linc-KILH on HCC cells was dependent on KRT19 expression. Furthermore, we conducted a series of rescue experiments, and the results further validated the necessity of KRT19 for the promoting function of Linc-KILH in HCC cells.

In conclusion, we validate Linc-KILH as a tumor promoting lncRNA, which is up-regulated in HCC tissues and could act as a molecular regulator of KRT19 to heighten Notch1 signaling, and in turn enhances the growth and metastasis abilities of KRT19 positive HCC cells, consequently boosting the development of HCC. We also detailed the mechanism of Linc-KILH on the regulation of KRT19 and $\beta$-catenin subcellular localization. Our findings indicate that Linc-KILH exerts a vital effect in KRT19 positive HCC development and may likely be developed into an effective therapeutic target for HCC.

\section{Supplementary Material}

Supplementary figures and tables. http://www.ijbs.com/v17p0768s1.pdf

\section{Acknowledgements}

This study was supported by National Natural Science Foundation of China (81702323, 81672469 and 81702338), the Social Development Foundation of Science and Technology of Jiangsu (BE2016658), and the Changzhou Sci \&Tech Program (No. CE20165020).

\section{Author's Contributions}

QXH and ZCF designed the study. ZXD, XXL and ZZC collected the clinical data and performed the experiments. XCL, KZJ and WSY performed the animal study and data analysis. ZXD and XXL wrote the manuscript. ZZC, YX and FY revised the paper. All authors read and approved the final manuscript.

\section{Competing Interests}

The authors have declared that no competing interest exists.

\section{References}

1. Bray F, Ferlay J, Soerjomataram I, Siegel RL, Torre LA, Jemal A. Global cancer statistics 2018: GLOBOCAN estimates of incidence and mortality worldwide for 36 cancers in 185 countries. CA: a cancer journal for clinicians 2018;68:394-424.

2. Clark T, Maximin S, Meier J, Pokharel S, Bhargava P. Hepatocellular Carcinoma: Review of Epidemiology, Screening, Imaging Diagnosis, Response Assessment, and Treatment. Current problems in diagnostic radiology 2015;44:479-486.

3. Waghray A, Murali AR, Menon KN. Hepatocellular carcinoma: From diagnosis to treatment. World journal of hepatology 2015;7:1020-1029.

4. Asfaha S, Hayakawa Y, Muley A, Stokes S, Graham TA, Ericksen RE, et al. Krt19(+)/Lgr5(-) Cells Are Radioresistant Cancer-Initiating Stem Cells in the Colon and Intestine. Cell stem cell 2015;16:627-638.

5. Govaere O, Komuta M, Berkers J, Spee B, Janssen C, de Luca F, et al. Keratin 19: a key role player in the invasion of human hepatocellular carcinomas. Gut 2014;63:674-685.

6. Saha SK, Choi HY, Kim BW, Dayem AA, Yang GM, Kim KS, et al. KRT19 directly interacts with beta-catenin/RAC1 complex to regulate NUMBdependent NOTCH signaling pathway and breast cancer properties. Oncogene 2017;36:332-349. 
7. Saha SK, Yin Y, Chae HS, Cho SG. Opposing Regulation of Cancer Properties via KRT19-Mediated Differential Modulation of Wnt/beta-Catenin/Notch Signaling in Breast and Colon Cancers. Cancers 2019;11.

8. Fatourou E, Koskinas J, Karandrea D, Palaiologou M, Syminelaki T, Karanikolas M, et al. Keratin 19 protein expression is an independent predictor of survival in human hepatocellular carcinoma. European journal of gastroenterology \& hepatology 2015;27:1094-1102.

9. Kawai T, Yasuchika K, Ishii T, Katayama H, Yoshitoshi EY, Ogiso S, et al. Keratin 19, a Cancer Stem Cell Marker in Human Hepatocellular Carcinoma. Clinical cancer research : an official journal of the American Association for Cancer Research 2015;21:3081-3091.

10. Yao H, Yang Z, Liu Z, Miao X, Yang L, Li D, et al. Glypican-3 and KRT19 are markers associating with metastasis and poor prognosis of pancreatic ductal adenocarcinoma. Cancer biomarkers: section A of Disease markers 2016;17:397-404

11. Govaere O, Petz M, Wouters J, Vandewynckel YP, Scott EJ, Topal B, et al. The PDGFRalpha-laminin B1-keratin 19 cascade drives tumor progression at the invasive front of human hepatocellular carcinoma. Oncogene 2017;36:6605-6616.

12. Iyer MK, Niknafs YS, Malik R, Singhal U, Sahu A, Hosono Y, et al. The landscape of long noncoding RNAs in the human transcriptome. Nature genetics 2015;47:199-208.

13. Schmitt AM, Chang HY. Long Noncoding RNAs in Cancer Pathways. Cancer cell 2016;29:452-463.

14. Takahashi K, Yan I, Haga H, Patel T. Long noncoding RNA in liver diseases. Hepatology 2014;60:744-753.

15. Huarte $M$. The emerging role of lncRNAs in cancer. Nature medicine 2015;21:1253-1261.

16. Liu N, Liu Q, Yang X, Zhang F, Li X, Ma Y, et al. Hepatitis B VirusUpregulated LNC-HUR1 Promotes Cell Proliferation and Tumorigenesis by Blocking p53 Activity. Hepatology 2018;68:2130-2144.

17. Quinn JJ, Chang HY. Unique features of long non-coding RNA biogenesis and function. Nature reviews Genetics 2016;17:47-62.

18. Wang YL, Liu JY, Yang JE, Yu XM, Chen ZL, Chen YJ, et al. Lnc-UCID Promotes G1/S Transition and Hepatoma Growth by Preventing DHX9Mediated CDK6 Down-regulation. Hepatology 2019;70:259-275.

19. Wang $\mathrm{P}$, Xue $\mathrm{Y}, \mathrm{Han} \mathrm{Y}$, Lin L, Wu C, Xu S, et al. The STAT3-binding long noncoding RNA lnc-DC controls human dendritic cell differentiation. Science 2014;344:310-313.

20. Tsai MC, Manor O, Wan Y, Mosammaparast N, Wang JK, Lan F, et al. Long noncoding RNA as modular scaffold of histone modification complexes. Science 2010;329:689-693.

21. Li YP, Duan FF, Zhao YT, Gu KL, Liao LQ, Su HB, et al. A TRIM71 binding long noncoding RNA Trincr1 represses FGF/ERK signaling in embryonic stem cells. Nature communications 2019;10:1368.

22. Kim H, Choi GH, Na DC, Ahn EY, Kim GI, Lee JE, et al. Human hepatocellular carcinomas with "Stemness"-related marker expression: keratin 19 expression and a poor prognosis. Hepatology 2011;54:1707-1717.

23. Uenishi T, Kubo S, Yamamoto T, Shuto T, Ogawa M, Tanaka H, et al. Cytokeratin 19 expression in hepatocellular carcinoma predicts early postoperative recurrence. Cancer science 2003;94:851-857.

24. Kong L, Zhang Y, Ye ZQ, Liu XQ, Zhao SQ, Wei L, et al. CPC: assess the protein-coding potential of transcripts using sequence features and support vector machine. Nucleic acids research 2007;35:W345-349.

25. Wang L, Park HJ, Dasari S, Wang S, Kocher JP, Li W. CPAT: Coding-Potential Assessment Tool using an alignment-free logistic regression model. Nucleic acids research 2013;41:e74.

26. Ding $\mathrm{CH}$, Yin $\mathrm{C}$, Chen $\mathrm{SJ}$, Wen LZ, Ding $\mathrm{K}$, Lei SJ, et al. The HNF1alpharegulated IncRNA HNF1A-AS1 reverses the malignancy of hepatocellular carcinoma by enhancing the phosphatase activity of SHP-1. Molecular cancer 2018;17:63.

27. Hu Q, Li C, Wang S, Li Y, Wen B, Zhang Y, et al. LncRNAs-directed PTEN enzymatic switch governs epithelial-mesenchymal transition. Cell research 2019;29:286-304.

28. Ju JH, Oh S, Lee KM, Yang W, Nam KS, Moon HG, et al. Cytokeratin19 induced by HER2/ERK binds and stabilizes HER2 on cell membranes. Cell death and differentiation 2015;22:665-676.

29. Omary MB, Ku NO, Tao GZ, Toivola DM, Liao J. "Heads and tails" of intermediate filament phosphorylation: multiple sites and functional insights. Trends in biochemical sciences 2006;31:383-394.

30. Zhou X, Liao J, Hu L, Feng L, Omary MB. Characterization of the major physiologic phosphorylation site of human keratin 19 and its role in filament organization. The Journal of biological chemistry 1999;274:12861-12866.

31. Tang J, Zhuo H, Zhang X, Jiang R, Ji J, Deng L, et al. A novel biomarker Linc00974 interacting with KRT19 promotes proliferation and metastasis in hepatocellular carcinoma. Cell death \& disease 2014:5:e1549.

32. Mai H, Zhou B, Liu L, Yang F, Conran C, Ji Y, et al. Molecular pattern of IncRNAs in hepatocellular carcinoma. Journal of experimental \& clinical cancer research: CR 2019;38:198.

33. Orom UA, Shiekhattar R. Long noncoding RNAs usher in a new era in the biology of enhancers. Cell 2013;154:1190-1193.

34. Jiang R, Tang J, Chen $Y$, Deng L, Ji J, Xie $Y$, et al. The long noncoding RNA lnc-EGFR stimulates T-regulatory cells differentiation thus promoting hepatocellular carcinoma immune evasion. Nature communications 2017;8:15129.
35. Li JK, Chen C, Liu JY, Shi JZ, Liu SP, Liu B, et al. Long noncoding RNA MRCCAT1 promotes metastasis of clear cell renal cell carcinoma via inhibiting NPR3 and activating p38-MAPK signaling. Molecular cancer 2017;16:111.

36. Xu X, Lou $Y$, Tang J, Teng $Y$, Zhang $Z$, Yin $Y$, et al. The long non-coding RNA Linc-GALH promotes hepatocellular carcinoma metastasis via epigenetically regulating Gankyrin. Cell death \& disease 2019;10:86.

37. Zhang A, Zhou N, Huang J, Liu O, Fukuda K, Ma D, et al. The human long non-coding RNA-RoR is a p53 repressor in response to DNA damage. Cell research 2013;23:340-350.

38. Zhang J, Li Z, Liu L, Wang Q, Li S, Chen D, et al. Long noncoding RNA TSLNC8 is a tumor suppressor that inactivates the interleukin-6/STAT3 signaling pathway. Hepatology 2018;67:171-187. 